\title{
Análise das mudanças sócio, econômico e ambientais após a transição do manejo convencional para o orgânico na produção de hortifrutis em uma propriedade rural no município de Ouro Fino - MG
}

Eustáchio Carneiro ${ }^{1}$

\section{Resumo}

A produção integrada de horticultura e fruticultura costuma ser associada ao acesso a novos mercados, maior qualidade dos produtos, boas práticas agrícolas, redução do impacto ambiental e bem estar social. Visando compreender melhor essas possibilidades, este trabalho avaliou o impacto social, econômico e ambiental que a conversão do manejo convencional para o orgânico proporcionou a uma propriedade familiar rural em Ouro Fino-MG. Os dados para a avaliação foram colhidos em 2012 conforme as tabelas do Sistema AMBITEC-AGRO 1.2. Concluiu-se que a conversão do manejo convencional para o orgânico trouxe ganhos sociais, econômicos e ambientais; e que a degradação ambiental e os riscos de contaminação ambiental diminuíram consideravelmente. Acredita-se que a metodologia de avaliação empregada neste trabalho possa servir para mensurar os impactos sociais, econômicos e ambientais em áreas mais abrangentes, como regiões ou estados.

Palavras-chave: Agricultura orgânica. Sustentabilidade. Agroecologia.

\section{Introdução}

O Sistema AMBITEC - AGRO 1.2 é um esquema que pode ser utilizado para referenciar mudanças socioeconômicas e ambientais, durante e após sua adoção ou a modificação de uma prática de manejo ou trabalho agrícola, e permite demonstrar claramente as conquistas no sistema de produção adotado. 0 produtor em questão aderiu ao sistema orgânico de produção para atender aos interesses tanto dos consumidores quanto dos agricultores em termos de segurança alimentar, bemestar, proteção ambiental e saúde.

Segundo o Ministério da Agricultura, Pecuária e Abastecimento (MAPA, 2005, apud SABBAG, 2013), a fruticultura é uma das atividades agrícolas que mais cresce no agronegócio brasileiro. O grande potencial produtivo do país, favorecido pela diversidade de condições climáticas, permite a produção de vários tipos de frutas tropicais e temperadas. Por exemplo, com base em dados do IBGE (2004), observa-se que, no período de 1994 a 2004, a produção brasileira de abacaxi evoluiu de 1.106.960 toneladas para 3.113.464 toneladas. Este aumento resultou não só da expansão da área colhida, mas também do aumento da produtividade, que evoluiu de 32,435 t/ha para 49,295 t/ha.

A produção integrada de frutas e hortaliças adotada na propriedade estudada tem como objetivo produzir frutas de qualidade, priorizando a conservação dos recursos naturais e a segurança do produtor e do consumidor, adotando, para tanto, o manejo orgânico. A qualidade dos produtos agroalimentares, particularmente os consumidos in natura, pode afetar a saúde dos consumidores. É importante ressaltar, ainda, que a definição da qualidade do produto tem demandado a consideração dos procedimentos envolvidos nas várias etapas da produção, incluindo-se as atividades agrícolas, o processamento, a industrialização, o transporte, os serviços de comercialização e o consumo. Sabe-

1 Instituto Federal de Educação, Ciência e Tecnologia do Sul de Minas Gerais - campus Inconfidentes. dangoecarneiro@yahoo.com.br. Praça Tiradentes, n416- Centro - Inconfidentes - MG. CEP 37576-000 
se que a qualidade duvidosa de um produto pode trazer prejuízos à saúde do consumidor, além de diminuir a credibilidade de empresas e países exportadores, que podem vir a sofrer, dessa forma, restrições de vendas no mercado internacional (Oliveira, 2005).

Os benefícios gerados a partir da utilização de sistemas de produção que privilegiam a conservação da biodiversidade, embora pouco estudados e de difícil quantificação, representam um importante benefício social, tanto no aspecto de conservação ambiental quanto de qualidade de vida.

E é nesta dimensão que se encontra o principal aporte desse tipo de manejo para a competitividade do agronegócio (EMBRAPA, 2007). De acordo com Rodrigues, Campanhola e Kitamura (2003), a dimensão social é parte indissociável das metodologias desenvolvidas para a avaliação dos impactos provocados pelas inovações tecnológicas aplicadas às atividades produtivas em estabelecimentos rurais. Assim, é importante identificar como e onde as tecnologias interferem no processo de produção e, a partir daí, saber como provocam modificações em diferentes aspectos da organização social (QUIRINO, 1999), objetivando a mensuração da eficiência e o incremento do impacto social positivo (YEGANIANTZ; MACEDO, 2002).

A avaliação dos impactos sociais de tecnologias e manejo adotadas na realização das atividades produtivas rurais é a fase final da pesquisa e desenvolvimento, mas tão importante como as etapas de análise e de validação (TUPY et al., 2006). Não se pode chegar a uma avaliação sem antes analisar criteriosamente as etapas de transição, que possivelmente gera pontos positivos e negativos.

0 presente estudo teve como objetivo utilizar o aplicativo Ambitec-Agro 1.2 para avaliar os impactos sociais promovidos pela transição do manejo convencional de frutas e hortaliças para 0 manejo orgânico em uma propriedade familiar no município de Ouro Fino - MG.

\section{Material e métodos}

O estudo foi realizado no ano de 2013 numa propriedade rural com base na agricultura familiar no município de Ouro Fino - MG e trata da avaliação do impacto social, econômico e ambiental provocado pela transição de manejo convencional para o orgânico no cultivo de frutas e hortaliças. 0 estudo foi realizado através de abordagem ao produtor e observação em campo, em uma propriedade agrícola familiar que dispõe de uma área de 12 ha, produzindo principalmente frutas e hortaliças, onde atualmente possui a certificação da Associação Natural de Campinas (ANC), o produtor é membro da Associação Agroecológica de Ouro Fino (AAOF) e escoa sua produção em gôndolas de produtos orgânicos em supermercados do município de Ouro Fino - MG e aos finais de semana na feira de agricultura orgânica em Campinas - SP.

O Sistema de Avaliação de Impacto Social de Inovações Tecnológicas Agropecuárias (Ambitec-Agro) empregado neste estudo consiste de um conjunto de quatorze indicadores explicativos dos impactos sociais, econômicas e ambientais, resultantes de uma dada atividade agrícola âmbito de um estabelecimento familiar rural. Esses indicadores são agrupados em quatro aspectos de consideração, quais sejam: Emprego, Renda, Saúde e Gestão e Administração.

A avaliação com o Sistema envolveu duas etapas: a primeira refere-se ao processo de levantamento e coleta de dados gerais sobre a tecnologia (abrangência e influência) e a segunda etapa trata das avaliações de campo e entrevistas individuais com os responsáveis pela adoção da prática adequada sobre os impactos avaliados nos diferentes indicadores. Os índices de impacto são obtidos em procedimentos de ponderação por fatores relativos à escala da ocorrência da alteração e ao peso dos componentes do indicador.

O fator de ponderação para escala de ocorrência do componente explicita o espaço no qual ocorre a alteração no componente do indicador, conforme a situação específica de aplicação da tecnologia, e pode ser: 1) Pontual, quando o efeito da tecnologia no componente restringe-se ao campo de cultivo ou unidade produtiva na qual esteja ocorrendo a alteração (fator de ponderação 1); 2) Local, quando o efeito se faz sentir externamente a essa unidade produtiva, porém confinado aos limites da propriedade (fator de ponderação 2); 3) No entorno, quando o efeito abrange além dos limites da propriedade (fator de ponderação 5). O fator de ponderação da escala da ocorrência implica a multi- 
plicação do coeficiente de alteração do componente por um valor predeterminado. 0 segundo fator de ponderação incluída nas matrizes de avaliação de impacto da inovação tecnológica é a importância do componente para a formação do indicador de impacto ambiental.

O sistema de pontuação utilizado reflete a avaliação sensorial sobre a alteração do indicador, a alteração refere-se ao desempenho da atividade desenvolvida com a inovação tecnológica, em comparação à atividade desenvolvida antes da adoção. Para um grande aumento no componente 0 valor da pontuação é $(+3)$; para um moderado aumento no componente é $(+1)$; quando componente inalterado é (0); para uma moderada diminuição é (-1) e quando ocorre grande diminuição é (-3). São atribuídos, ainda, pesos para os componentes, que variam conforme a sua quantidade na formação do indicador, sendo, portanto, usados para normalizar os indicadores conforme o teste de sensibilidade aplicado caso a caso (GIRARDIN et al., 1999). Os pesos dos componentes expressos nas matrizes podem ser alterados pelo usuário do sistema para melhor refletir situações específicas de avaliação, nas quais se pretenda enfatizar alguns dos componentes, desde que o peso total dos componentes para um dado indicador seja igual à unidade (1). Cabe ressaltar que para uma mesma tecnologia, mesmo quando utilizada em diferentes sistemas naturais, os valores dos pesos dos componentes dos indicadores devem permanecer os mesmos, a fim de que os resultados sejam comparáveis. Os resultados finais da avaliação de impacto são expressos graficamente, com os indicadores considerados em seu conjunto para composição do Índice de Impacto Social da Inovação Tecnológica.

A escala padronizada no sistema varia entre -15 e +15, normalizada para todos os indicadores individualmente e para o Índice Geral de Impacto Social da Tecnologia. É importante esclarecer que o método traz como norma de avaliação a adequação tecnológica definida como minimização de impactos negativos, em qualquer dos indicadores. Para maiores detalhes sobre o Sistema AmbitecAgro ver Rodrigues, Campanhola e Kitamura (2003).

\section{Resultados e discussão}

O trabalho objeto do estudo foi realizado em janeiro de 2013 em propriedade rural familiar com área de 12 ha, a qual é administrada pelo proprietário que ali cultiva hortifruticultura orgânica.

Verificaram-se as alterações ocorridas referentes ao uso de insumos agrícolas como os citados abaixo. Houve uma drástica diminuição do uso de insumos agrícolas como pesticidas e fertilizantes químicos, uma vez que a prática de manejo orgânico não prevê a utilização dos mesmos. Consequentemente houve uma diminuição considerável do uso de água, já que a maioria dos pesticidas outrora utilizados necessitava de água. Esse é um forte fator econômico e ambiental a favor do manejo orgânico (Quadro 1).

\begin{tabular}{|c|c|c|c|c|c|c|c|c|}
\hline \multirow{3}{*}{\multicolumn{3}{|c|}{$\begin{array}{l}\text { Uso de Insumos } \\
\text { Agricolas e Recursos }\end{array}$}} & \multirow{2}{*}{\multicolumn{3}{|c|}{$\begin{array}{c}\text { observadas no consumo de insumos e recurso } \\
\text { Variáveis de us o de insumos }\end{array}$}} & \multirow{2}{*}{\multicolumn{2}{|c|}{$\begin{array}{l}\text { Vs, POR UNIDADE DE PRODUTC } \\
\text { Variáveis de uso de recurs os } \\
\text { naturais }\end{array}$}} & \multirow{3}{*}{$\begin{array}{l}\text { Averiguação } \\
\text { fatores de } \\
\text { ponderação }\end{array}$} \\
\hline & & & & & & & & \\
\hline & & & \multirow{2}{*}{$\begin{array}{c}\text { Pesticidas } \\
-0,4 \\
\end{array}$} & \multirow{2}{*}{$\begin{array}{c}\text { Fertilizantes } \\
-0,25\end{array}$} & $\begin{array}{c}\text { Condicionadores } \\
\text { de solo }\end{array}$ & \multirow{2}{*}{$\begin{array}{c}\text { Consumo de } \\
\text { água }\end{array}$} & \multirow{2}{*}{$\begin{array}{c}\text { Solo (área) } \\
-0,1\end{array}$} & \\
\hline Fato & s de ponder & ão $\mathrm{k}$ & & & $-0,15$ & & & \multirow[t]{5}{*}{-1} \\
\hline \multirow{4}{*}{ 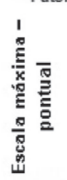 } & $\begin{array}{c}\text { Não se } \\
\text { aplica }\end{array}$ & $\begin{array}{c}\text { Marcar } \\
\infty \mathrm{m} X\end{array}$ & & & & & & \\
\hline & Pontual & 5 & -3 & -3 & & -3 & 1 & \\
\hline & Local & - & ........... & $\ldots \ldots \ldots .$. & $\ldots \ldots \ldots . .$. & ........... & $\ldots \ldots \ldots . .$. & \\
\hline & Entorno & - & $\ldots \ldots \ldots .$. & $\ldots \ldots \ldots .$. & $\ldots$ & $\ldots \ldots \ldots .$. & $\ldots \ldots \ldots . .$. & \\
\hline \multicolumn{3}{|c|}{$\begin{array}{l}\text { Coeficiente de impacto }= \\
\text { (coeficientes de alteração * } \\
\text { fatores de ponderação) }\end{array}$} & 6 & 3,75 & 0 & 1,5 & $-0,5$ & 10,75 \\
\hline
\end{tabular}

Quadro 1. Alterações observadas na propriedade relativas ao consumo de insumos e recursos. Fonte: Elaboração dos autores

Com relação à qualidade do solo observou-se, após a transição do sistema convencional para o orgânico, uma diminuição dos pontos com potencial erosivo devido à escassez de cobertura vegetal e sua consequente perda de nutrientes e compactação, visto que as práticas orgânicas protegem a massa vegetal e o uso de capinas ao invés de herbicidas sistêmicos e de contato ajuda a preservá-la, aumentando, assim, sua atividade microbiológica. (Quadro 2). 


\begin{tabular}{|c|c|c|c|c|c|c|c|}
\hline \multicolumn{8}{|c|}{ Que alteraçốes foram observadas na qualidade do solo? } \\
\hline \multirow{2}{*}{\multicolumn{3}{|c|}{ Qualidade do Solo }} & \multicolumn{4}{|c|}{ Variáveis de qualidade do solo } & \multirow{2}{*}{$\begin{array}{l}\text { Averiguação } \\
\text { fatores de } \\
\text { ponderação }\end{array}$} \\
\hline & & & Erosão & $\begin{array}{l}\text { Perda de matéria } \\
\text { orgânica }\end{array}$ & $\begin{array}{l}\text { Perda de } \\
\text { nutrientes }\end{array}$ & Compactação & \\
\hline \multicolumn{3}{|c|}{ Fatores de ponderação $k$} & $-0,25$ & $-0,25$ & $-0,25$ & $-0,25$ & \multirow[t]{5}{*}{-1} \\
\hline \multirow{4}{*}{ 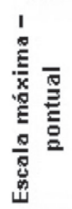 } & $\begin{array}{c}\text { Não se } \\
\text { aplica }\end{array}$ & $\begin{array}{l}\text { Marcar } \\
\infty \mathrm{m} \mathrm{X}\end{array}$ & & & & & \\
\hline & Pontual & 5 & -3 & -3 & -3 & -3 & \\
\hline & Local & - & $\ldots \cdots \cdots$ & $\ldots \cdots \cdots$ & $\ldots \cdots \cdots$ & $\ldots \cdots \cdots$ & \\
\hline & Entorno & - & $\ldots \ldots \ldots$ & $\ldots \ldots \ldots . .$. & ……...... & $\ldots \ldots \ldots .$. & \\
\hline \multicolumn{3}{|c|}{$\begin{array}{c}\text { Coeficiente de impacto }= \\
\text { (coeficientes de alteração } \\
\text { fatores de ponderação) }\end{array}$} & 3,75 & 3,75 & 3,75 & 3,75 & 15,00 \\
\hline
\end{tabular}

Quadro 2. Alterações observadas na qualidade do solo da propriedade em análise.

Fonte: Elaboração do autor

Outro fator ambiental visto nesse aspecto foi a diminuição da carga orgânica lançada nos cursos de água e a diminuição do assoreamento, fato possível graças à diminuição da lixiviação do solo promovida pela manutenção da cobertura vegetal (Quadro 3).

\begin{tabular}{|c|c|c|c|c|c|c|c|}
\hline \multirow{3}{*}{\multicolumn{3}{|c|}{ Qualidade da Água }} & am observadas & qualidade & água? & & \multirow[b]{3}{*}{$\begin{array}{l}\text { Averiguação } \\
\text { fatores de } \\
\text { ponderação }\end{array}$} \\
\hline & & & \multicolumn{4}{|c|}{ Variáveis de qualidade da água } & \\
\hline & & & \multicolumn{2}{|l|}{\begin{tabular}{|c|} 
Carga \\
orgânica \\
(efluentes, \\
esgotos, \\
estercos, etc.)
\end{tabular}} & $\begin{array}{l}\text { Espumas / Óleos } \\
\text { / Resíduos } \\
\text { sólidos }\end{array}$ & $\begin{array}{c}\text { Assoreamento } \\
\text { de corpos } \\
\text { d'água }\end{array}$ & \\
\hline \multicolumn{3}{|c|}{ Fatores de ponderação $k$} & $-0,25$ & $-0,25$ & $-0,25$ & $-0,25$ & \multirow[t]{5}{*}{-1} \\
\hline \multirow{4}{*}{ 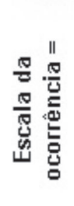 } & $\begin{array}{c}\text { Não se } \\
\text { aplica }\end{array}$ & $\begin{array}{c}\text { Marcar } \\
\operatorname{com~X}\end{array}$ & & & & & \\
\hline & Pontual & 1 & -3 & 0 & -3 & -3 & \\
\hline & Local & 2 & -3 & 0 & -1 & -3 & \\
\hline & Entorno & 5 & -3 & 0 & 0 & 0 & \\
\hline \multicolumn{3}{|c|}{$\begin{array}{l}\text { Coeficiente de impacto }= \\
\text { (coeficientes de alteração } \\
\text { fatores de ponderação) }\end{array}$} & 6 & 0 & 1,25 & 2,25 & 9,50 \\
\hline
\end{tabular}

Quadro 3. Alterações observadas na qualidade da água da propriedade.

Fonte: Elaboração do autor

Sobre a recuperação dos ambientes degradados, verificou-se considerável recomposição natural das áreas de preservação permanente bem como o atendimento à legislação quanto à reserva legal, pois a propriedade possui hoje mais de $20 \%$ de sua área com cobertura vegetal, o que acarretou a diminuição de solos e ecossistemas degradados e áreas sujeitas à erosão (Quadro 4).

\begin{tabular}{|c|c|c|c|c|c|c|c|}
\hline \multicolumn{8}{|c|}{ Que alteraçöes foram observadas na recuperação de ambientes degradados? } \\
\hline \multirow{2}{*}{\multicolumn{3}{|c|}{$\begin{array}{c}\text { Recuperação } \\
\text { Ambiental }\end{array}$}} & \multicolumn{4}{|c|}{ Variáveis de recuperação ambiental } & \multirow[b]{2}{*}{$\begin{array}{l}\text { Averiguação } \\
\text { fatores de } \\
\text { ponderação }\end{array}$} \\
\hline & & & $\begin{array}{c}\text { Solos } \\
\text { degradados }\end{array}$ & $\begin{array}{c}\text { Ecossistemas } \\
\text { degradados }\end{array}$ & $\begin{array}{l}\text { Áreas de } \\
\text { Preservação } \\
\text { Permanente }\end{array}$ & Reserva Legal & \\
\hline \multicolumn{3}{|c|}{ Fatores de ponderação $k$} & 0,2 & 0,2 & 0,2 & 0,4 & \multirow[t]{5}{*}{1} \\
\hline \multirow{4}{*}{ 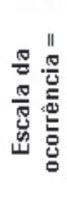 } & $\begin{array}{l}\text { Não se } \\
\text { aplica }\end{array}$ & $\begin{array}{l}\text { Marcar } \\
\infty \mathrm{m} \mathrm{X}\end{array}$ & & & & $\mathrm{x}$ & \\
\hline & Pontual & 1 & 3 & 3 & 3 & 0 & \\
\hline & Local & 2 & 3 & 1 & 3 & 3 & \\
\hline & Entorno & 5 & 3 & 0 & 3 & & \\
\hline \multicolumn{3}{|c|}{$\begin{array}{l}\text { Coeficiente de impacto = } \\
\text { (coeficientes de alteraçãa * } \\
\text { fatores de ponderação) }\end{array}$} & 4,8 & 1 & 4,8 & 2,4 & 13,00 \\
\hline
\end{tabular}

Quadro 4. Alterações observadas na recuperação de ambientes degradados.

Fonte: Elaboração do autor 


\section{Aspectos Socioeconômicos}

Abordando os aspectos socioeconômicos verifica-se um aumento da estabilidade de mercado e local de distribuição dos produtos após certificação orgânica e adesão à associação de produtores (Quadro 5).

\begin{tabular}{|c|c|c|c|c|c|c|c|}
\hline & ue altera & es fora & obs ervadas & as variáveis de & eração de rer & & \\
\hline & & & & Atributos & a renda & & \\
\hline Ger & ção de F & nda & $\begin{array}{c}\text { Segurança } \\
\text { (garantia de } \\
\text { obteção) }\end{array}$ & $\begin{array}{l}\text { Estabilidade } \\
\text { (sazonalidade) }\end{array}$ & Distribuição & Montante & $\begin{array}{l}\text { fatores de } \\
\text { ponderação }\end{array}$ \\
\hline Fato & s de ponder & ão $k$ & 0,25 & 0,25 & 0,25 & 0,25 & 1 \\
\hline$\stackrel{1}{E}$ & $\begin{array}{c}\text { Não se } \\
\text { aplica }\end{array}$ & $\begin{array}{l}\text { Marcar } \\
\text { com X }\end{array}$ & & & & & \\
\hline 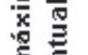 & Pontual & 5 & 1 & 3 & 3 & 3 & \\
\hline$\frac{1}{0}$ & Local & - & ............ & . & $\ldots$ & ........... & \\
\hline 总 & Entorno & - & ........... & (n........ & .............. & .......... & \\
\hline $\begin{array}{r}\text { Coefi } \\
\text { (coefic } \\
\text { fator }\end{array}$ & $\begin{array}{l}\text { ente de im } \\
\text { ntes de alt } \\
\text { de ponde }\end{array}$ & $\begin{array}{l}\text { acto = } \\
\text { ação * } \\
\text { çã̃o) }\end{array}$ & 1,25 & 3,75 & 3,75 & 3,75 & 12,50 \\
\hline
\end{tabular}

Quadro 5. Alterações observadas em função das variáveis geradoras de renda.

Fonte: Elaboração do autor

Outro fator percebido no quadro 6 é a diminuição de riscos quanto à emissão poluentes tanto na atmosfera quanto no solo e que podem ser carreados para os cursos d'água.

\begin{tabular}{|c|c|c|c|c|c|c|c|c|}
\hline \multicolumn{9}{|c|}{ Que alteraçóes foram obs ervadas nas variáveis de saúde ambiental e pes soal? } \\
\hline \multirow{2}{*}{\multicolumn{3}{|c|}{$\begin{array}{c}\text { Saúde Ambiental e } \\
\text { Pessoal }\end{array}$}} & \multicolumn{5}{|c|}{ Variável de s aúde amb iental e pessoal } & \multirow[b]{2}{*}{$\begin{array}{l}\text { Averiguação } \\
\text { fatores de } \\
\text { ponderação }\end{array}$} \\
\hline & & & $\begin{array}{l}\text { Focos de } \\
\text { vetores de } \\
\text { doenças } \\
\text { endêmicas }\end{array}$ & $\begin{array}{l}\text { Emissão de } \\
\text { poluentes } \\
\text { atmosféricos }\end{array}$ & $\begin{array}{l}\text { Emissão de } \\
\text { poluentes } \\
\text { hídricos }\end{array}$ & $\begin{array}{c}\text { Geração de } \\
\text { contaminantes } \\
\text { do solo }\end{array}$ & $\begin{array}{c}\text { Dificuldade de } \\
\text { acesso a } \\
\text { esporte e } \\
\text { lazer } \\
\end{array}$ & \\
\hline \multicolumn{3}{|c|}{ Fatores de ponderação $k$} & $-0,2$ & $-0,2$ & $-0,2$ & $-0,2$ & $-0,2$ & \multirow[t]{5}{*}{-1} \\
\hline \multirow{4}{*}{ 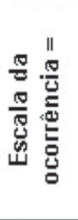 } & $\begin{array}{l}\text { Não se } \\
\text { aplica }\end{array}$ & $\begin{array}{l}\text { Marcar } \\
\text { com X }\end{array}$ & $\mathrm{x}$ & & & & $\mathrm{x}$ & \\
\hline & Pontual & 1 & -3 & -3 & -3 & -3 & & \\
\hline & Local & 2 & -1 & -3 & -3 & -3 & & \\
\hline & Entorno & 5 & -1 & -1 & -1 & -1 & & \\
\hline \multicolumn{3}{|c|}{$\begin{array}{l}\text { Coeficiente de impacto }= \\
\text { (coeficientes de alteração * } \\
\text { fatores de ponderação) }\end{array}$} & 2 & 2,8 & 2,8 & 2,8 & 0 & 10,40 \\
\hline
\end{tabular}

Quadro 6. Alterações observadas relativas às variáveis de saúde ambiental e pessoal

Fonte: Elaboração do autor

Já no quesito segurança alimentar, tem-se um aumento da qualidade nutricional do alimento, que é cultivado sem adição de nenhum agrotóxico ou adubo químico, estabelecendo uma relação de cooperação entre solo sadio e cultivo. É importante salientar aqui que com o avanço de técnicas de manejo agrícola sustentável existe a capacidade de produção em uma escala considerável segura para que não haja, após o período de transição, a queda no volume de produção (Quadro 7). 


\begin{tabular}{|c|c|c|c|c|c|c|}
\hline \multirow{3}{*}{\multicolumn{3}{|c|}{ Segurança Alimentar }} & obs ervadas & s variáveis d & egurança alim & ntar? \\
\hline & & & \multicolumn{3}{|c|}{ Variáveis de segurança alimentar } & \multirow[b]{2}{*}{$\begin{array}{c}\text { Averiguação } \\
\text { fatores de } \\
\text { ponderação }\end{array}$} \\
\hline & & & \multirow{3}{*}{$\begin{array}{c}\begin{array}{c}\text { Garantia da } \\
\text { produção }\end{array} \\
0,3 \\
\end{array}$} & \multirow{3}{*}{$\begin{array}{c}\text { Quantidade } \\
\text { de alimento }\end{array}$} & \multirow{2}{*}{$\begin{array}{c}\begin{array}{c}\text { Qualidade } \\
\text { nutricional do } \\
\text { alimento }\end{array} \\
0,4 \\
\end{array}$} & \\
\hline \multirow{5}{*}{ 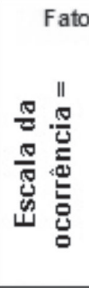 } & es de ponder & ão $k$ & & & & \multirow[t]{5}{*}{1} \\
\hline & $\begin{array}{c}\text { Não se } \\
\text { aplica }\end{array}$ & $\begin{array}{l}\text { Marcar } \\
\text { com X }\end{array}$ & & & & \\
\hline & Pontual & 1 & 0 & 3 & 3 & \\
\hline & Local & 2 & 0 & 3 & 3 & \\
\hline & Entorno & 5 & 0 & 1 & 3 & \\
\hline \multicolumn{3}{|c|}{$\begin{array}{c}\text { Coeficiente de impacto = } \\
\text { (coeficientes de alteração * } \\
\text { fatores de ponderação) }\end{array}$} & 0 & 4,2 & 9,6 & 13,80 \\
\hline
\end{tabular}

Quadro 7. Alterações observadas na propriedade relativas às variáveis de segurança alimentar. Fonte: Elaboração do autor

Em relação ao perfil profissional, constatou-se que durante o processo de implantação do sistema orgânico a promoção do trabalho familiar dentro da unidade de produção foi importante e significativa, diminuindo custos com pessoal, atraindo a maior parte da renda para dentro da unidade. Há também melhorias no quesito de capacitação que o próprio sistema orgânico exige e que é possível de se conseguir através de vínculo com cooperativas e associações de agricultores (Quadro 8).

\begin{tabular}{|c|c|c|c|c|c|c|c|c|c|}
\hline \multicolumn{10}{|c|}{ Que alterações foram obs ervadas nas variáveis de ded ic ação e de perfil profissional do res pons ável? } \\
\hline \multirow{2}{*}{\multicolumn{3}{|c|}{$\begin{array}{c}\text { Dedicação e Perfil do } \\
\text { Responsável }\end{array}$}} & \multicolumn{6}{|c|}{ Variáveis de dedicação do res ponsável } & \multirow[b]{2}{*}{$\begin{array}{l}\text { Averiguação } \\
\text { fatores de } \\
\text { ponderação }\end{array}$} \\
\hline & & & $\begin{array}{l}\text { Capacitação } \\
\text { dirigida à } \\
\text { atividade }\end{array}$ & $\begin{array}{c}\text { Horas de } \\
\text { permanência no } \\
\text { estabelecimento }\end{array}$ & $\begin{array}{l}\text { Engajamento } \\
\text { familiar }\end{array}$ & $\begin{array}{l}\text { Uso de siste ma } \\
\text { contábil }\end{array}$ & $\begin{array}{l}\text { Modelo formal } \\
\text { de } \\
\text { planejamento }\end{array}$ & $\begin{array}{l}\text { Sistema de } \\
\text { certificação / } \\
\text { Rotulagem }\end{array}$ & \\
\hline \multicolumn{3}{|c|}{ Fatores de ponderaçãa $\mathrm{k}$} & 0,2 & 0,2 & 0,15 & 0,15 & 0,15 & 0,15 & \multirow[t]{5}{*}{1} \\
\hline \multirow{4}{*}{ 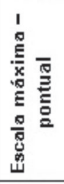 } & $\begin{array}{l}\text { Não se } \\
\text { aplica }\end{array}$ & $\begin{array}{l}\text { Marcar } \\
\operatorname{com~X~}\end{array}$ & & & & & & & \\
\hline & Pontual & 5 & 3 & 3 & 3 & 1 & 1 & 3 & \\
\hline & Local & - & $\ldots \ldots \ldots$ & $\ldots \ldots \ldots$ & $\ldots \ldots \ldots$ & $\ldots \ldots$ & $\ldots \ldots \ldots$ & $\ldots \ldots \ldots$ & \\
\hline & Entorno & - & ........... & ........... & ........... & ............ & $\ldots \ldots \ldots$ & $\ldots \ldots \ldots$ & \\
\hline \multicolumn{3}{|c|}{$\begin{array}{l}\text { Coeficiente de impacto = } \\
\text { (coeficientes de alteração * } \\
\text { fatores de ponderaçẫo) }\end{array}$} & 3 & 3 & 2,25 & 0,75 & 0,75 & 2,25 & 12,00 \\
\hline
\end{tabular}

Quadro 8. Variações observadas relativas à dedicação e ao perfil profissional dos responsáveis pela propriedade. Fonte: Elaboração do autor

Ainda avaliando as mudanças socioeconômicas, é possível visualizar um aumento na capacidade de venda, já que o produtor faz parte de uma associação que lhe dá suporte para conquista de mercado, garantindo assim o engajamento de seu produto nas cadeias de comércio (Quadro 9).

\begin{tabular}{|c|c|c|c|c|c|c|c|c|c|c|}
\hline \multicolumn{11}{|c|}{ Que alterações foram obs ervadas nas variáveis de comercialização? } \\
\hline \multirow{2}{*}{\multicolumn{3}{|c|}{$\begin{array}{c}\text { Condição de } \\
\text { Comercialização }\end{array}$}} & \multicolumn{7}{|c|}{ Variáveis de comercialização } & \multirow[b]{2}{*}{$\begin{array}{l}\text { Averiguaçãa } \\
\text { fatores de } \\
\text { ponderação }\end{array}$} \\
\hline & & & $\begin{array}{l}\text { Venda direta / } \\
\text { antecipada / } \\
\text { cooperada }\end{array}$ & $\begin{array}{l}\text { Processamento } \\
\text { local }\end{array}$ & $\begin{array}{c}\text { Amazenamento } \\
\text { local }\end{array}$ & $\begin{array}{c}\text { Transporte } \\
\text { próprio }\end{array}$ & $\begin{array}{l}\text { Propaganda / } \\
\text { Marca própria }\end{array}$ & $\begin{array}{c}\text { Encadeamento } \\
\text { com produtos / } \\
\text { atividades / } \\
\text { serviços } \\
\text { anteriores }\end{array}$ & $\begin{array}{c}\text { Cooperação } \\
\text { com outros } \\
\text { produtores } \\
\text { locais }\end{array}$ & \\
\hline \multicolumn{3}{|c|}{ Fatores de ponderação $k$} & 0,15 & 0,15 & 0,15 & 0,15 & 0,15 & 0,15 & 0,1 & \multirow[t]{5}{*}{1} \\
\hline \multirow{4}{*}{ 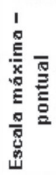 } & $\begin{array}{l}\text { Não se } \\
\text { aplica }\end{array}$ & $\begin{array}{l}\text { Marcar } \\
\text { com X }\end{array}$ & & & & & & & & \\
\hline & Pontual & 5 & 3 & 3 & 3 & 3 & 3 & 1 & 3 & \\
\hline & Local & - & ........... & …........ & ........... & …........ & …....... & …....... & ............ & \\
\hline & Entorno & - & ............. & ............. & ................ & ................ & ............... & ……........ & ................ & \\
\hline \multicolumn{3}{|c|}{$\begin{array}{l}\text { Coeficiente de impacto }= \\
\text { (coeficientes de alteração * } \\
\text { fatores de ponderação) }\end{array}$} & 2,25 & 2,25 & 2,25 & 2,25 & 2,25 & 0,75 & 1,5 & 13,50 \\
\hline
\end{tabular}

Quadro 9. Alterações observadas relativas às variáveis de comercialização de produtos da propriedade. Fonte: Elaboração do autor 
Na tabela abaixo, verifica-se um aumento no reaproveitamento de resíduos orgânicos, provenientes de outras atividades na propriedade, diminuindo assim os impactos negativos ao meio ambiente quanto à disposição de resíduos (Quadro 10).

\begin{tabular}{|c|c|c|c|c|c|c|c|c|}
\hline \multicolumn{9}{|c|}{ Que alterações foram obs ervadas nas variáveis de dispos ição de resíduos? } \\
\hline \multirow{2}{*}{\multicolumn{3}{|c|}{$\begin{array}{l}\text { Disposição de } \\
\text { Residuos }\end{array}$}} & \multicolumn{3}{|c|}{ Tratamento de res iduos domés ticos } & \multicolumn{2}{|c|}{$\begin{array}{c}\text { Tratamento de res íduos da } \\
\text { produção }\end{array}$} & \multirow{2}{*}{$\begin{array}{l}\text { Averiguação } \\
\text { fatores de } \\
\text { ponderação }\end{array}$} \\
\hline & & & Coleta seletiva & $\begin{array}{l}\text { Compostagem / } \\
\text { Reaproveitamento }\end{array}$ & $\begin{array}{l}\text { Disposição } \\
\text { sanitária }\end{array}$ & $\begin{array}{l}\text { Reaproveita- } \\
\text { mento }\end{array}$ & $\begin{array}{c}\text { Destinação } \\
\text { ou tratamento } \\
\text { final }\end{array}$ & \\
\hline \multicolumn{3}{|c|}{ F atores de ponderação $k$} & 0,2 & 0,2 & 0,2 & 0,2 & 0,2 & \multirow[t]{5}{*}{1} \\
\hline \multirow{4}{*}{ 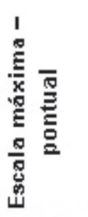 } & $\begin{array}{l}\text { Não se } \\
\text { aplica }\end{array}$ & $\begin{array}{l}\text { Marcar } \\
\operatorname{com} X\end{array}$ & & & & & & \\
\hline & Pontual & 5 & 0 & 3 & 3 & 3 & 3 & \\
\hline & Local & - & $\ldots \ldots \ldots$ & $\ldots \ldots \ldots$ & $\ldots \ldots \ldots$ & $\ldots \ldots \ldots$ & $\ldots \ldots \ldots$ & \\
\hline & Entorno & - & …...... & $\ldots \ldots \ldots$ & …....... & …...... & $\ldots \ldots \ldots$ & \\
\hline \multicolumn{3}{|c|}{$\begin{array}{l}\text { Coeficiente de impacto = } \\
\text { (coeficientes de alteração * } \\
\text { fatores de ponderação) }\end{array}$} & 0 & 3 & 3 & 3 & 3 & 12,00 \\
\hline
\end{tabular}

Quadro 10. Alterações observadas na propriedade em função da disposição de resíduos.

Fonte: Elaboração do autor

Um dos fatores mais importantes durante o processo de transição e a escolha pelo manejo orgânico é a abordagem que se vê no quadro abaixo, com relação a insumos químicos. A utilização de produtos químicos para o cultivo de frutas e hortaliças foi totalmente retirada da propriedade, houve uma preocupação em inserir na propriedade o uso contínuo de equipamentos de proteção individual, para todos que participam de qualquer etapa de produção. Houve também um investimento em um local adequado para armazenamento de embalagens que já foram utilizadas, como sacos de adubo orgânico, por exemplo (Quadro 11).

\begin{tabular}{|c|c|c|c|c|c|c|c|c|}
\hline \multicolumn{9}{|c|}{ Que alterações foram obs ervadas nas variáveis de ges tão de ins umos químicos? } \\
\hline \multirow{2}{*}{\multicolumn{3}{|c|}{$\begin{array}{c}\text { Gestão de Insumos } \\
\text { Químicos }\end{array}$}} & \multicolumn{5}{|c|}{ Variáveis de gestão de insumos químicos } & \multirow[b]{2}{*}{$\begin{array}{l}\text { Averiguação } \\
\text { fatores de } \\
\text { ponderação }\end{array}$} \\
\hline & & & $\begin{array}{l}\text { Armazena- } \\
\text { mento }\end{array}$ & $\begin{array}{c}\text { Calibração e } \\
\text { verificação de } \\
\text { equipamentos } \\
\text { de aplicação }\end{array}$ & $\begin{array}{l}\text { Utilização de } \\
\text { equipamentos } \\
\text { de proteção } \\
\text { individual }\end{array}$ & $\begin{array}{l}\text { Disposição final } \\
\text { adequada de } \\
\text { recipentes e } \\
\text { embalagens }\end{array}$ & $\begin{array}{l}\text { Registro dos } \\
\text { tratamentos }\end{array}$ & \\
\hline \multicolumn{3}{|c|}{ Fatores de ponderação $\mathrm{k}$} & 0,2 & 0,25 & 0,25 & 0,15 & 0,15 & \multirow[t]{5}{*}{1} \\
\hline \multirow{4}{*}{ 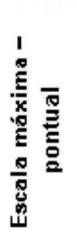 } & $\begin{array}{l}\text { Não se } \\
\text { aplica }\end{array}$ & $\begin{array}{c}\text { Marcar } \\
\text { com X }\end{array}$ & & & & & & \\
\hline & Pontual & 5 & 3 & 3 & 3 & 3 & 3 & \\
\hline & Local & - & $\ldots+\cdots \cdots \cdots$ & ................... & ................... & .................. & ..................... & \\
\hline & Entorno & - & ....................... & ..................... & ...................... & ................... & ..................... & \\
\hline \multicolumn{3}{|c|}{$\begin{array}{c}\text { Coeficiente de impacto }= \\
\text { (coeficientes de alteração * } \\
\text { fatores de ponderação) }\end{array}$} & 3 & 3,75 & 3,75 & 2,25 & 2,25 & 15,00 \\
\hline
\end{tabular}

Quadro 11. Alterações observadas em função da gestão de insumos químicos na propriedade estudada.

Fonte: Elaboração do autor

Quando estudado o relacionamento institucional, destaca-se o quesito associativismo e a utilização de recursos legais. A propriedade deu um grande salto após se associar a um grupo de produtores com o mesmo interesse de produção e mercado. Assim, alcançaram certificação, buscam capacitação e meios de melhoria da vida no campo, sendo este um fator que merece muita atenção quando se pensa na necessidade da diminuição do êxodo rural, agregação de valor ao produto agrícola e melhoria de condições para que o produtor consiga produzir em uma escala maior produtos saudáveis e de qualidade (Quadro 12). 


\begin{tabular}{|c|c|c|c|c|c|c|c|c|c|}
\hline \multicolumn{10}{|c|}{ Que alteraçőes foram obs ervadas nas variáveis de relacionamento institucional? } \\
\hline \multirow{2}{*}{\multicolumn{3}{|c|}{$\begin{array}{l}\text { Relacionamento } \\
\text { Institucional }\end{array}$}} & \multicolumn{4}{|c|}{ Variáveis de alcance institucional } & \multicolumn{2}{|c|}{$\begin{array}{c}\text { Variáveis de capacitação } \\
\text { contínua }\end{array}$} & \multirow{2}{*}{$\begin{array}{l}\text { Averiguação } \\
\text { fatores de } \\
\text { ponderação }\end{array}$} \\
\hline & & & $\begin{array}{l}\text { Utilização de } \\
\text { assistência } \\
\text { técnica }\end{array}$ & $\begin{array}{l}\text { Associativismo / } \\
\text { Cooperativismo }\end{array}$ & $\begin{array}{l}\text { Filiação } \\
\text { tecnológica } \\
\text { nominal }\end{array}$ & $\begin{array}{l}\text { Utilização de } \\
\text { assessoria } \\
\text { legal / Vistoria }\end{array}$ & Gerente & $\begin{array}{c}\text { Empregados } \\
\text { especializados }\end{array}$ & \\
\hline \multicolumn{3}{|c|}{ Fatores de ponderação $\mathrm{k}$} & 0,2 & 0,2 & 0,15 & 0,15 & 0,15 & 0,15 & \multirow[t]{5}{*}{1} \\
\hline \multirow{4}{*}{ 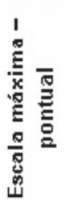 } & $\begin{array}{l}\text { Não se } \\
\text { aplica }\end{array}$ & $\begin{array}{c}\text { Marcar } \\
\operatorname{com~X}\end{array}$ & & & & & & & \\
\hline & Pontual & 5 & 3 & 3 & 1 & 3 & 3 & 0 & \\
\hline & Local & - & ............... & $\ldots \ldots \ldots . . . .$. & $\ldots \ldots \ldots . . .$. & $\ldots \ldots \ldots$ & ............... & ................ & \\
\hline & Entorno & - & $\ldots \ldots \ldots . .$. & $\ldots \ldots \ldots$ & $\ldots \ldots \ldots$ & $\ldots$ & $\ldots \ldots \ldots . .$. & $\ldots \ldots \ldots$ & \\
\hline \multicolumn{3}{|c|}{$\begin{array}{l}\text { Coeficiente de impacto }= \\
\text { (coeficientes de alteração * } \\
\text { fates de ponderacão) }\end{array}$} & 3 & 3 & 0,75 & 2,25 & 2,25 & 0 & 11,25 \\
\hline
\end{tabular}

Quadro 12. Alterações observadas relativas às variáveis de relacionamento institucional.

Fonte: Elaboração do autor

\section{Conclusões}

Diante das observações e registros realizados, conclui-se que:

1) 0 processo de avaliação Ambitec-Agro permite validar essas informações de uma maneira de fácil e claro entendimento.

2) A análise do impacto ambiental apresenta como benefício a eliminação da aplicação de agroquímicos e uma melhoria substancial no uso do solo.

3) O sistema orgânico de produção, após implantado, contribuiu para a minimização de impactos ambientais, sobressaindo-se no processo de comercialização para o mercado externo em função das exigências dos mercados compradores para a cultura de frutas e hortaliças.

4) Em função do crescimento do mercado consumidor, verifica-se a necessidade de ampliação da área de produção. Sendo este um fator econômico considerável e passivo de estudo, no que diz respeito à agregação e à elevação de renda, possibilitando a sustentabilidade em todas as dimensões.

\section{Analysis of social, economic and environmental changes after the transition from conventional to organic management in the production of vegetables and fruit on a farm in Ouro Fino - MG}

\section{Abstract}

The integrated production of horticultural and fruit is always associated with the access to new markets, higher quality products, good agricultural practices, reducing environmental impact and improving social welfare. To better understand these possibilities, this work evaluated the social, economic and environmental impact after the transition from conventional to organic management in horticulture and fruit production in a rural family property in Ouro Fino, Minas Gerais. To carry out this work and to evaluate the data, collected during the year of 2012, System Ambitec-AGRO 1.2 tables were used. The conclusion was that the changing to the organic management has brought social, economic and environmental gains and that the environmental degradation and the risks of contamination decreased so much. It is believed that this method of measuring the gains brought about by that changing can be used to evaluate the social, economic and environmental impacts in broader areas, like regions and states.

Keywords: Organic agriculture. Sustainability. Agroecology. 


\section{Referências bibliográficas}

EMBRAPA. Produção Integrada de Maçãs no Brasil. 2007. Disponível em <http://sistemasdeproducao.cnptia.embrapa.br/FontesHTML/Maca/ProducaolntegradaMaca/index.htm>. Acesso em: 15 jan. 2013.

GIRARDIN, P.; BOCKSTALLER, C.; WERF, H. van der. Indicators: tools to evaluate the environmental impacts of farming systems. J. Sustain. Agric., Binghamton, v. 13, n. 4, p. 5-21, 1999.

INSTITUTO BRASILEIRO DE GEOGRAFIA E ESTATÍSTICA (IBGE). Censo 2004. Disponível em: <http:/www.ibge.gov.br>. Acesso em: 23 jan. 2013.

OLIVEIRA, L. Antunes. A importância das normas internacionais para o comércio da fruticultura brasileira. 2005, 168 f. Dissertação (Mestrado), Universidade de São Paulo, Economia Aplicada, Piracicaba, 2005.

QUIRINO, T. R.; IRIAS, L. J. M.; WRIGHT, J. T. C. Impacto agroambiental - perspectivas, problemas e prioridades. São Paulo: Edgard Blücher, 1999. 184 p

RODRIGUES, G. S., CAMPANHOLA, C., KITAMURA, P. C. Avaliação de impacto ambiental da inovação tecnológica agropecuária: Ambitec-Agro. Jaguariúna: Embrapa Meio Ambiente, 2003. (Documentos, 34).

SABBAG, O. J. Avaliação de impactos ambientais pós-certificação eurepgap na cultura do abacaxi em guaraçaí (SP). Disponível em <http://www.revistas.ufg.br/index.php/pat/article/view/3904>. Acesso em: 12 jan. 2013.

TUPY, O. et al. Avaliação dos impactos econômicos, sociais e ambientais de tecnologias da Embrapa Pecuária Sudeste. São Carlos: Embrapa Pecuária Sudeste, 2006. (Documentos, 08).

YEGANIANTZ, L.; MACEDO, M. M. C. Avaliação de impacto social de pesquisa agropecuária: a busca de uma metodologia baseada em indicadores. Brasília: Embrapa Informação Tecnológica, 2002. (Texto para Discussão; 13).

Histórico editorial

Recebido: 17/05/2013

Avaliação e copidesque: 27/05/2013 a 24/10/2013 